\title{
ECG Signal Classification for Remote Area Patients Using Artificial Neural Networks in Smartphone
}

\author{
Vincent D. Montaño ${ }^{1 *}$, Noel B. Linsangan², Noel D. Ramos ${ }^{2}$ \\ ${ }^{1}$ College of Engineering, Samar State University, Catbalogan City, Samar, Philippines. \\ 2 Computer Engineering Department, Mapúa Institute of Technology, Intramuros, Manila, Philippines. \\ * Corresponding author. Tel.: (063) 917590 8465; email: vincent.montano@ssu.edu.ph \\ Manuscript submitted August 1, 2014; accepted April 10, 2015. \\ doi: 10.17706/ijcee.2015.7.3.215-222
}

\begin{abstract}
Heart disease is one of the main causes of global death, and instant diagnosis of this condition is significant for health improvement. This condition can be classified using the electrocardiogram (ECG) signal information. Application of artificial neural network (ANN) as a medical diagnostic classifier has been suggested by various studies in signal recognition. Collaboration with the recent advances in mobile technology for processing and transmission of medical data where medical feedback can be delivered promptly. This study presents a method of ECG signal classification for the patient coming from remote areas using ANN modeled algorithm used in a smartphone. The system is composed of monitoring device that accepts ECG signal from the patient and transmit this signal through a General Packet Radio Service (GPRS) technology. And a smartphone that receives and processes the information for medical ECG classification. Five type of ECG signals obtained from the selected arrhythmia database were classified with the sensitivity of $96.67 \%$, specificity of $99.17 \%$ and correctness rate of $98.67 \%$ by the proposed method.
\end{abstract}

Key words: Algorithm, artificial neural network (ANN), electrocardiogram (ECG), general packet radio service (GPRS), smartphone.

\section{Introduction}

Heart diseases remain as one of the leading causes of deaths over the years, not only in the country but also globally as well. According to the data recorded by the Department of Health (DOH) from 2004 to 2009, 21 percent of all deaths in the country are due to heart diseases. One of these diseases is arrhythmia, the unusual or abnormal activity of the heart. The American Heart Association (AHA) stated that the term "arrhythmia" refers to any change from the normal sequence of electrical impulses. The electrical impulses may happen too fast, too slowly, or erratically - causing the heart to beat too fast, too slowly, or erratically. A physical diagnostic test used for classifying cardiac arrhythmia is the application of electrocardiogram. AHA standards also defined electrocardiogram (ECG) as a test that measures the electrical activity of the heartbeat. With each beat, an electrical impulse (or "wave") travels through the heart. This wave causes the muscle to squeeze and pump blood from the heart. By determining the information present in an ECG data, various forms of cardiac arrhythmias can be identified. Consequently, ECG serves a vital part in detecting and classifying irregularities in the rhythm of the heart rate by calculating the path of electrical output of the heart. Therefore, the classification of ECG data is of considerable importance for prevention, treatment and proper diagnosis of heart conditions.

In the past years, numerous approaches have been suggested in the classification of ECG signals. Many 
study proposed various method of algorithm for classification and feature extractions of ECG signals [1]-[6]. One of those focused on the application of cutting-edge technology and computational algorithm for grouping of cardiac arrhythmia [7]. The proposed algorithm used the application of fuzzy method and extended fuzzy for automated detection, classification and clustering of cardiac arrhythmia. Another study used a combined neural network model based on stacked generalization method for classification of ECG beats [8]. In the conventional method, the combiner learns to map the base classifier outputs to the target data. The study concluded that by combining the original input data to the output, and as the result, by increasing the awareness of the combiner, the algorithm helped it making a better decision according to the base classifier decisions. Artificial neural network (ANN) has been used in a huge amount of medical diagnostic classifier. By incorporating interconnected layers, this computational model mimics some properties of biological neurons. Thus, significant parameters are found from complex relationship between inputs and outputs.

Various study about remote monitoring and diagnosis utilized different technology like smartphone and GPS [9], [10]. One of those is the application of mobile technology and cloud computing that inspired various designs of health monitoring system [11]. In this cloud-based system, a real time ECG monitoring and analysis was developed with an algorithm for ECG enhancement, extraction and evaluation. Another study about a real-time cardiovascular detection using ECG processing [12]. The paper seek to unite the portability of Holter monitors and the real-time processing capability of state-of-the-art resting ECG machines to provide an assistive diagnosis solution using smartphones.

Integration between these modeled algorithms with the evolving mobile technology would enhance the medical analysis and portability of bio signal classification particularly in ECG signal.

Previous studies have addressed the problem of heart rate and cardiac arrhythmia monitoring. Based in the study of Albert et al. regarding the monitoring system of the heart using a smartphone or computer, the researchers developed a personal physiology monitoring device for delivering ECG and cardiac arrhythmia [13]. The developed device was capable of monitoring physiological signals from the patient through electrode attached to the skin and on the smartphone. In another study, the researchers developed a mobile remote monitoring system to enable walking-based rehabilitation in which the patient's ECG and other information is transferred by a smartphone for real-time monitoring [14]. Although numerous studies have been explored and invented for the monitoring of ECG signals, detection and proper classification of this ECG signal through a flexible model is required for preventive diagnosis of heart related diseases as well as portability in monitoring using mobile devices which is essential for the development of a remote health care system in the country.

The study created a monitoring device for acquisition and transmission of ECG information from the patient. Developed a mobile application as a graphical user interface (GUI) to display the ECG diagnosis. Designed an artificial neural network algorithm for classification of ECG information using the data from the MIT-BIH (Massachusetts Institute of Technology - Beth Israel Hospital) Arrhythmia Database. Integrated the monitoring device to the mobile application in the smartphone and analyze the modeled ANN algorithm for the accuracy of the developed system for ECG classification using the data from the MIT-BIH.

\section{Methodology}

The methodology of the study involves the hardware design, software design and the artificial neural network information for acquisition and classification of ECG information.

\subsection{Hardware Development}

Fig. 1 shows the component of the developed device. The proposed hardware used the Arduino Mega 2560 as the microcontroller. It can be used to create independent device and can be connected to the 
software on a computer. For the GSM/GPRS module, a physical add-on shield compatible to the microcontroller was used. The Gizmo GSM/GPRS Shield connected the Arduino board device to the internet using the GPRS network architecture. The Olimex EKG/EMG shield was used which allows microcontroller to capture electrocardiography-electromyography signals. The SHIELD-EKG-EMG is an extension module for Olimex's ARDUINO compatible boards. Besides, for the LCD module, the system used a typical $16 \times 2$ LCD as monitor display in the device.

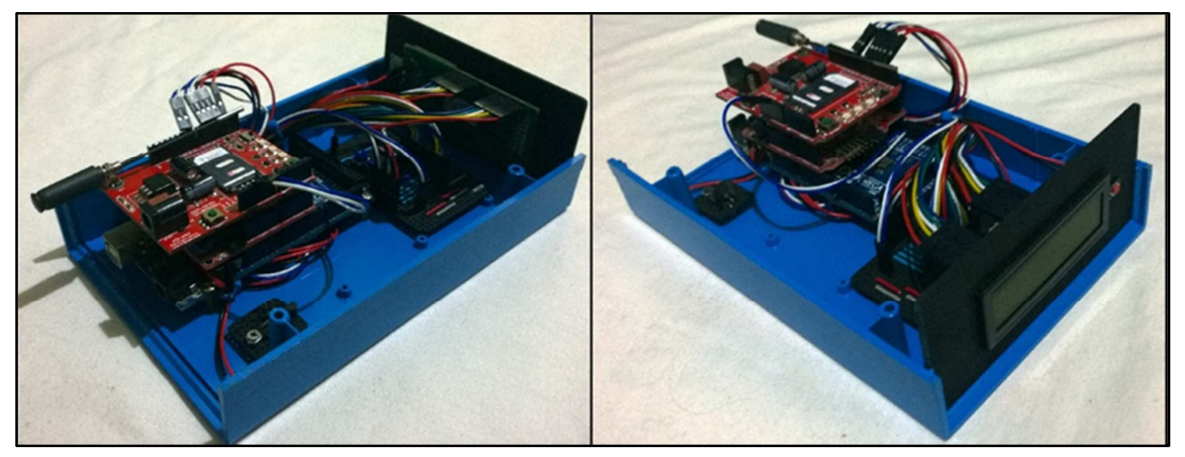

Fig. 1. Developed hardware device.

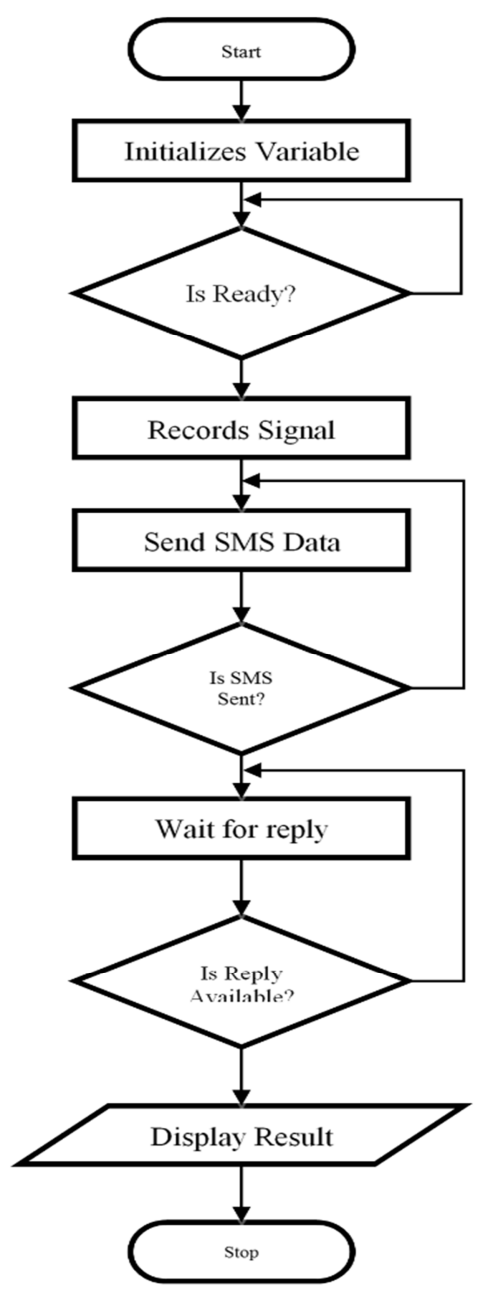

Fig. 2. Hardware device flowchart.

Fig. 2 shows the flowchart of the program execution in the monitoring device. Initialization of variables and data as performed before waiting from the response of the user. Then the program executed the 
recording of ECG signal for 8 seconds. The data recorded were transmitted through SMS then wait for the reply to be displayed in the device.

\subsection{Software Development}

Table 1 shows the elements required for the development of the mobile application. C\# programming language was used as the main language of the mobile program and as the controller of the logic in the program code-behind. Moreover, extensible application markup language (XAML) acts as the declarative markup language for simplified user interface (UI) framework creation.

Table 1. Mobile Application Development

\begin{tabular}{cc}
\hline \hline \multicolumn{2}{c}{ Mobile Application Development } \\
\hline Programming Language & C\# \\
Markup Language & XAML \\
Mobile Platform & Windows Phone 8 \\
Integrated Development Environment & Visual Studio \\
(IDE) & 2012 \\
Cloud Computing Platform & Microsoft Azure \\
Mobile User Interface Components & Telerik DevTools \\
\hline \hline
\end{tabular}

Fig. 3 displays the software development process. The mobile software of the proposed system decrypted the received information from the monitoring device via the GPRS network. It performs an initial processing for verification and validation of the received data. The application program would execute a feature extraction from the data in preparation for ANN application. After the data are fed into the trained algorithm, the result of the processed information was available for display on the monitor of the smartphone. To support in creating the desired mobile application, the study provides a detailed specification of software tools to be used.

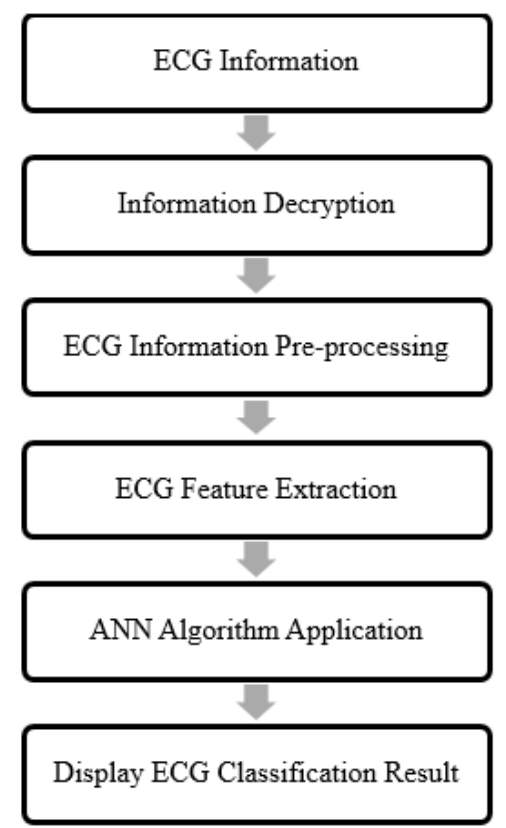

Fig. 3. Software development process.

\subsection{Artificial Neural Network Information}

The ANN Algorithm created using a C\# programming language applied multiple layers to transform data to create a new representation. The 1st layer used step activation transform with bias and holds 12 neurons. 
The 2nd layer used hyperbolic tangent activation with bias and holds 13 neurons. And the last layer used sigmoid function activation without bias and holds 5 neurons. The network is trained using a Levenberg Marquardt algorithm (LMA) with a training error of 0.0001 .

The normalization and selection of testing and training data are controlled by the Encog workbench using the Encog Analyst. This workbench is a GUI application that simulates the performance of most machine learning functions. Encog Analyst is a module in the Encog framework that executes a series of command for analyzing data.

Table 2 indicates the target vector in binary classification selected for the training of the ANN Algorithm. Each type of signal is classified to a specific binary representation to be used as a reference result of the proposed method. Type S contains atrial premature, nodal premature and supraventricular premature. Type $\mathrm{V}$ contains premature ventricular contraction and ventricular ectopic. Type $\mathrm{F}$ contains fusion of ventricular and normal beats. Type $Q$ contains paced beat and unclassified beat. And Type $\mathrm{N}$ includes normal beat, atrial escape, nodal escape, right and left bundle branch block. The output data served as the output neuron of the ANN Algorithm.

Table 2. Target Classification for ANN Algorithm

\begin{tabular}{|c|c|c|}
\hline Class & Type & Classification \\
\hline 1 & $S$ & 10000 \\
\hline 2 & $\mathrm{~F}$ & 01000 \\
\hline 3 & V & 00100 \\
\hline 4 & Q & 00010 \\
\hline 5 & $\mathrm{~N}$ & 000001 \\
\hline
\end{tabular}

\section{Results and Discussion}

In order to assess the performance and precision of the proposed method in distinguishing the ECG signal, the overall sensitivity (Se), specificity (Sp), and general correctness rate (GCR) are determined for the sample data from the MIT-BIH arrhythmia database.

The MIT-BIH Arrhythmia Database contains 48 half-hour excerpts of two-channel ambulatory ECG recordings, obtained from 47 subjects studied by the BIH Arrhythmia Laboratory between 1975 and 1979. Twenty-three recordings were chosen at random from a set of 4000 24-hour ambulatory ECG recordings collected from a mixed population of inpatients (about 60\%) and outpatients (about 40\%) at Boston's Beth Israel Hospital; the remaining 25 recordings were selected from the same set to include less common but clinically significant arrhythmias that would not be well-represented in a small random sample. The recordings were digitized at 360 samples per second per channel with 11-bit resolution over a $10 \mathrm{mV}$ range. Two or more cardiologists independently annotated each record; disagreements were resolved to obtain the computer-readable reference annotations for each beat (approximately 110,000 annotations in all) included with the database [15].

The ECG recognition algorithm is based on the vital parameters stored on the signal. The definitions of True Positive (TP, the number of true signal that has been correctly identified), False Positive (FP, the number of true signal that has been incorrectly identified), True Negative (TN, the number of false signal that has been successfully identified), and False Negative (FN, the count of false signal that has been incorrectly identified) are basis for the classification of an application. Accordingly, application of the below parameters is vital in examining the recognition algorithm.

$$
\text { Sensitivity, } \mathrm{Se}=\frac{\mathrm{TP}}{\mathrm{TP}+\mathrm{FN}} \%
$$




$$
\begin{aligned}
& \text { Specificity, } \mathrm{Sp}=\frac{\mathrm{TN}}{\mathrm{TN}+\mathrm{FP}} \% \\
& \mathrm{GCR}=\frac{\mathrm{TP}+\mathrm{TN}}{\mathrm{TP}+\mathrm{FN}+\mathrm{TN}+\mathrm{FP}} \%
\end{aligned}
$$

Table 3 displays the classification result of selected MIT-BIH files using the ANN Algorithm. The first row and the first column match with to the labels from the AAMI. Based from the result, Type S, Type V and Type $\mathrm{Q}$ performed better than the other types due to the unique distinction and characteristics of the said types. On the other hand, the result of Type $\mathrm{F}$, Type $\mathrm{V}$ and Type $\mathrm{N}$ were lower than the other. Due to the resemblance of Type $\mathrm{F}$ to Type $\mathrm{V}$ and Type $\mathrm{N}$, it is very complex to distinguish the features of this type.

Table 3. Summarized Recognition Result of Selected Dataset by ANN Algorithm

\begin{tabular}{c|ccccc}
\hline \hline & Type S & Type F & Type V & Type Q & Type N \\
\hline Type S & 30 & 0 & 0 & 0 & 0 \\
Type F & 0 & 25 & 1 & 0 & 4 \\
Type V & 0 & 0 & 30 & 0 & 0 \\
Type Q & 0 & 0 & 0 & 30 & 0 \\
Type N & 0 & 0 & 0 & 0 & 30 \\
\hline \hline
\end{tabular}

Table 4 shows the performance analysis of the classification result. To demonstrate the efficiency of the proposed algorithm for ECG detection, the following performance evaluation parameters are needed to evaluate the result of ECG arrhythmia classification. Sensitivity is the ability to correctly detect one class. Specificity is the ability to correctly detect the other category. And correctness refers to the ability to correctly recognize both categories (the general classification rate). Using the formula mentioned, the proposed classification performs with an average sensitivity of $96.67 \%$, specificity of $99.17 \%$ and general correctness rate of $98.67 \%$.

Table 4. Performance Analysis Result of the ECG Signal Classification

\begin{tabular}{cccc}
\hline \hline Arrhythmia Type & Sensitivity (\%) & Specificity (\%) & $\begin{array}{c}\text { Correctness } \\
(\%)\end{array}$ \\
\hline Type S & 100 & 100 & 100 \\
Type F & 83.33 & 100 & 96.67 \\
Type V & 100 & 99.17 & 99.33 \\
Type Q & 100 & 100 & 100 \\
Type N & 100 & 96.67 & 97.33 \\
\hline AVERAGE & 96.67 & 99.17 & 98.67 \\
\hline \hline
\end{tabular}

\section{Conclusion and Future Work}

A monitoring device was created using Arduino Mega 2560, Olimex EKG/EMG shield, and Gizmo GSM/GPRS module for acquisition and transmission of ECG information from the patient. A mobile application was developed using Visual Studio 2012 and Telerik RadControls as a graphical user interface (GUI) to display the ECG diagnosis. An artificial neural network algorithm was designed using the Encog library for classification of ECG information using the data from the MIT-BIH (Massachusetts Institute of Technology - Beth Israel Hospital) Arrhythmia Database. Five types of cardiac arrhythmia namely: Type S, Type V, Type F, Type $\mathrm{Q}$ and Type $\mathrm{N}$ were used for the basis of classification following the standard of The Association for the Advancement of Medical Instrumentation (AAMI). The monitoring device was integrated 
to the mobile application in the smartphone using the GPRS and Microsoft Azure infrastructure. And based from the statistical data analysis on the modeled ANN algorithm, the developed system performed with a sensitivity of $96.67 \%$, specificity of $99.17 \%$ and general correctness rate of $98.67 \%$.

The researcher recommends the development of the mobile application on other mobile platform like iOS and Android platform, perform signal amplification on the ECG signal to remove external noise on the data, provide display of the ECG signal on the device during recording of data, and perform SMS encryption to minimize the number of character in the SMS transferred.

\section{Acknowledgement}

V. M. Montaño would like to extend his deepest gratitude and appreciation to the following people for their invaluable help and support:

First and foremost, to God, for giving wisdom and perseverance; for the guidance in helping surpass all the trials that the author encountered and for giving determination to make this study possible.

To his alma mater, the Samar State University and Mapúa Institute of Technology for molding him into the person he is today.

To his family, for giving support and encouragement to pursue his study; for giving trust, love and patience.

To the School of Graduate Studies, for the financial support of his entire study under the ERDT Scholarship Program of the DOST.

Mr. Noel Linsangan and Mr. Noel Ramos, for giving invaluable assistance, support and supervision.

Finally, V. M. Montaño would like to thank his fiancée, April Aner, for the support through the duration of his studies.

\section{References}

[1] Bhyri, C., Hamde, S., \& Waghmare, L. (2011). ECG feature extraction and disease diagnosis. Journal of Medical Engineering \& Technology, 35, 354-361.

[2] Köhler, B., Hennig, C., \& Orglmeister, R. (2002). The principles of software QRS detection. IEEE Engineering in Medicine And Biology Magazine: The Quarterly Magazine of The Engineering in Medicine \& Biology Society, 21, 42-57.

[3] Martis, R., Acharya, U., Mandana, K. M., Ray, A. K., \& Chakraborty, C. (2012). Application of principal component analysis to ECG signals for automated diagnosis of cardiac health. Expert Systems With Applications, 14, 11792.

[4] Sayadi, O. O., Shamsollahi, M. B., \& Clifford, G. D. (2010). Robust detection of premature ventricular contractions using a wave-based Bayesian framework. IEEE Transactions on Bio-Medical Engineering, 57, 353-362.

[5] Pablo, L., Raimon, J., \& Pere, C. (1994). Automatic detection of wave boundaries in multi-lead ECG signals: Validation with the CSE database. Computers and Biomedical Research, 27, 45.

[6] Benali, R. R., Dib, N. N., \& Bereksi, F. F. R. (2010). Cardiac arrhythmia diagnosis using a neuro-fuzzy approach. Journal of Mechanics in Medicine and Biology, 10, 417-429.

[7] Mehdi, A., Zayegh, A., Begg, R., \& Ali, R. (2010). GK based fuzzy clustering for the diagnosis of cardiac arrhythmia. International Journal of Computational Intelligence and Applications, 9, 105-123.

[8] Javadi, M., Ebrahimpour, R., Sajedin, A., Faridi, S., \& Zakernejad, S. (2011). Improving ECG classification accuracy using an ensemble of neural network modules. PLOS One, 6.

[9] Dong, J., Zhang, J., Zhu, H., Wang, L., Liu, X., \& Li, Z. (2012). A remote diagnosis service platform for wearable ECG monitors. IEEE Intelligent Systems, 27, 36-43. 
[10] Worringham, C., Rojek, A., \& Stewart, I. (2011). Development and feasibility of a smartphone, ECG and GPS based system for remotely monitoring exercise in cardiac rehabilitation. PLOS One, 6, 1-6.

[11] Xia, H., Asif, I., \& Zhao, X. (2013). Cloud-ECG for real time ECG monitoring and analysis. Computer Methods and Programs in Biomedicine, 110, 253-259.

[12] Oresko, J. J., Jin, Z., Cheng, J., Huang, S., Sun, Y., Duschl, H., \& Cheng, A. C. (2010). A wearable smartphone-based platform for real-time cardiovascular disease detection via electrocardiogram processing. IEEE Transactions on Information Technology in Biomedicine, 14, 734-740.

[13] Albert, D., Satchwell, B. R., \& Barnett, K. N. (2011). Heart monitoring system usable with a smartphone or computer. USPTO Patent Applications.

[14] Hsieh, J., Shen, T., Lo, H., Gu, B., \& Chang, P. (2012). Mobile information system for 12-lead ECG. USPTO Patent Applications.

[15] Moody, G. B., \& Mark, R. G. (2002). The impact of the MIT-BIH arrhythmia database. IEEE Eng in Med and Biol., 20(3), 45-50.

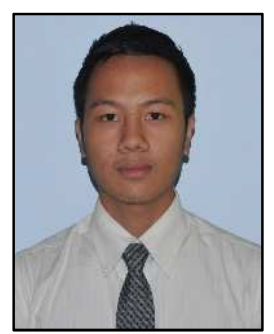

Vincent D. Montaño is a faculty member of the College of Engineering (COE) at the Samar State University (SSU), Catbalogan City, Samar, Philippines. He received his bachelor of science degree in computer engineering in March 2010 from this institution. Then received his master of science degree in computer engineering in July 2014 from the Mapúa Institute of Technology, Manila, Philippines under the Engineering Research and Development for Technology (ERDT) scholarship funded by the Department of Science and Technology (DOST). His research interests are mainly in biomedical engineering, object oriented programming, artificial intelligence, and machine learning.

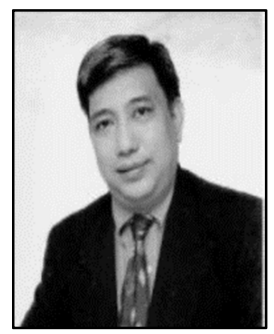

Noel B. Linsangan is the current program chair of the Computer Engineering ( $\mathrm{CpE}$ ) program at the Mapúa Institute of Technology, Manila, Philippines. He received his baccalaureate degree in computer engineering from the same school in 1988 and his master's degree of engineering in computer engineering from the Pamantasan ng Lungsodng Maynila, University of the City of Manila in 2000.

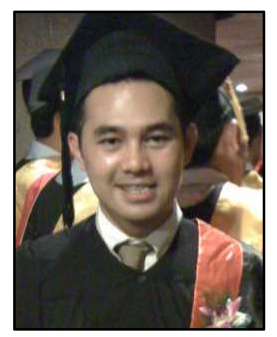

Noel D. Ramos is an information technology (IT) consultant at the Asian Development Bank (ADB), Mandaluyong, Philippines and a faculty member of the Computer Engineering Department in Mapúa Institute of Technology, Manila, Philippines. He finished his baccalaureate degree in computer engineering and the master of engineering degree in computer engineering in 2011 from the same institution. 